\title{
Descattering Transmission via Angular Filtering
}

\author{
Jaewon $\mathrm{Kim}^{1,2}$, Douglas Lanman ${ }^{1, \star}$, \\ Yasuhiro Mukaigawa ${ }^{1, \star \star}$, and Ramesh Raskar ${ }^{1}$ \\ 1 MIT Media Lab \\ ${ }^{2}$ Korea Institute of Science and Technology(KIST)
}

\begin{abstract}
We describe a single-shot method to differentiate unscattered and scattered components of light transmission through a heterogeneous translucent material. Directly-transmitted components travel in a straight line from the light source, while scattered components originate from multiple scattering centers in the volume. Computer vision methods deal with participating media via $2 \mathrm{D}$ contrast enhancing software techniques. On the other hand, optics techniques treat scattering as noise and use elaborate methods to reduce the scattering or its impact on the direct unscattered component. We observe the scattered component on its own provides useful information because the angular variation is low frequency. We propose a method to strategically capture angularly varying scattered light and compute the unscattered direct component. We capture the scattering from a single light source via a lenslet array placed close to the image plane. As an application, we demonstrate enhanced tomographic reconstruction of scattering objects using estimated direct transmission images.
\end{abstract}

Keywords: computational photography, direct transmission, scattered transmission, multiple scattering, image decomposition.

\section{Introduction}

The separation of direct and scattered components of incident light is a challenging topic in computer vision and graphics, a task that is confounded by the complex behavior of light in participating media, e.g., reflection, refraction, and scattering in haze, underwater or in volumetric translucent objects. These complex characteristics of light are one of the main factors hindering an analytical solution for direct-scattered separation. For this reason, active coding methods have been proposed. Nayar et al. 1 project high-frequency patterns onto a reflective scene. Such active coding methods achieve accurate and robust separation. Narasimhan et al. 2] use structured light to estimate the 3-D shape of objects in scattering media, including diluted suspensions. $\mathrm{Gu}$ et al. 3 also use structured light, exploiting compressive sensing techniques, to decrease the data acquisition time. Atcheson et al. 4] estimate the 3-D shape of non-stationary

\footnotetext{
* Visiting from Brown University.

** Visiting from Osaka University.

K. Daniilidis, P. Maragos, N. Paragios (Eds.): ECCV 2010, Part I, LNCS 6311, pp. 86-99, 2010.

(C) Springer-Verlag Berlin Heidelberg 2010
} 

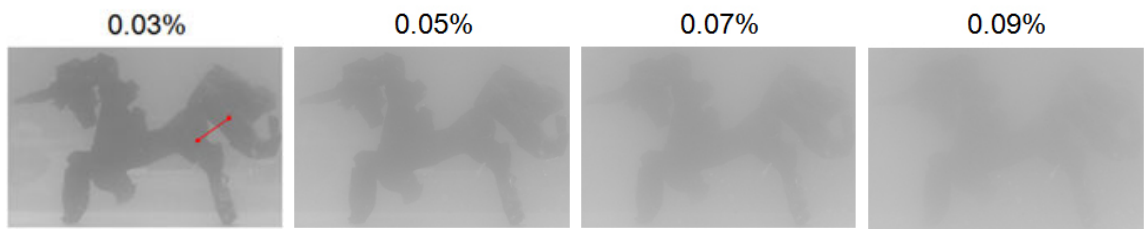

(a) Normal Photo
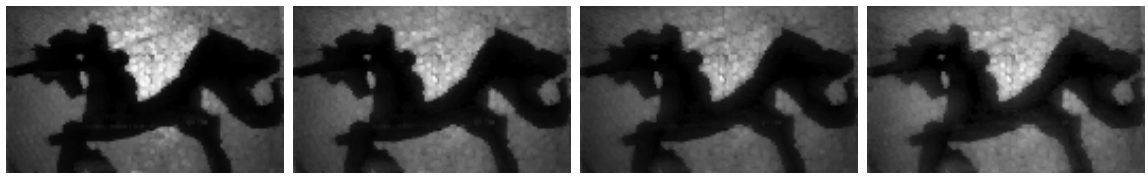

(b) Direct-only Image
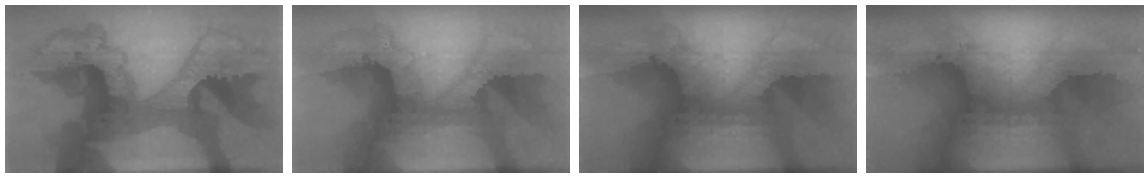

(c) Scattered-only Image
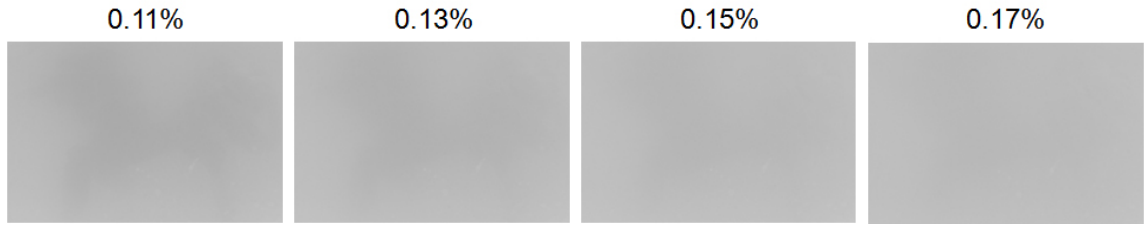

(d) Normal Photo
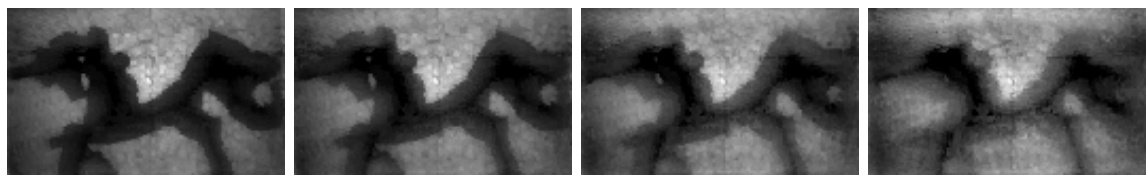

(e) Direct-only Image
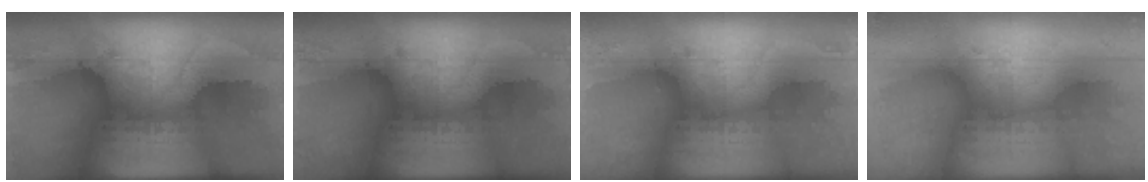

(f) Scattered-only Image

Fig. 1. Recovery of an opaque object in participating media with milky water. (a) and (d) Normal photos according to concentration $0.03 \%-0.17 \%$ in which water is $7500 \mathrm{ml}$ and milk is increased by $1.5 \mathrm{ml}$ from $2 \mathrm{ml}$. (b) and (e) Recovered direct-only images computed using angular-domain filtering with a lenslet array. Note enhanced visibility for sharp features and edges in the descattered image. (c) and (f) scattered-only images preprocessed to acquire the direct-only images. 
gas flows. In many existing approaches, only scattering scenes composed of low density materials (eg. smoke, liquid, and powder) are allowed, such that a single scattering mode is dominant. Using the methods outlined in this paper, we demonstrate direct-scattered separation for scenes in which multiple scattering is predominant.

In this paper we use a passive, single-shot imaging method to achieve separation of transmitted light for a scene containing heterogeneous scattering media. Specifically, we use a lenslet(or pinhole) array close to the image plane to separate direct and scattered components of incident light (albeit while reducing the resolution of the recovered direct and scattering component images since the projection of each lenslet or pinhole provides a single pixel in the recovered images). Using a sequence of such images, we are able to recover an estimate of the volumetric attenuation using existing tomographic reconstruction methods, demonstrating benefits for both dehazing and 3D shape recovery.

\subsection{Contributions}

We describe a method for single-exposure separation of direct and scattered components of transmitted light passing through scattering media using a lenslet or pinhole array placed closely to the image sensor. In the direct-only image, highfrequency details are restored and provide strong edge cues for scattering objects. Due to its single-shot nature, this method is well-suited for analyzing dynamic scenes. We demonstrate enhanced tomographic reconstruction of scattering objects using direct component images. These separation methods are well-suited for applications in medical imaging, providing an internal view of scattering objects such as human skin using visible or near-visible wavelength light sources, rather than X-rays.

\subsection{Related Work}

Direct-Scattered Separation: Direct-scattered separation of light is widely studied in diverse fields spanning computer vision, graphics, optics, and physics. Due to the complexities of scattering, reflection, and refraction, analytical methods do not achieve satisfactory results in practical situations. In computer vision and graphics, Nayar et al. 1 present an effective method to separate direct and scattered components from a scene by projecting a sequence of high-frequency patterns. Their work is one of the first to handle arbitrary natural scenes. However, it requires temporally-multiplexed illumination, limiting the utility for dynamic scenes. Nasu et al. [5] present an accelerated method using a sequence of three patterns. In addition, Rosen and Abookasis [6] present a descattering method using speckle analysis.

Microscopy: The scattering in microscopic objects is addressed by careful optical methods. Hisashi [7] present a method to achieve a sharp in-focus signal in a confocal microscope setup. They use two pinholes to sense in- and out-of-focus signals and acquire a sharp in-focus signal by subtracting the two. This requires two exposures and careful alignment for each spot. In addition, scanning process 


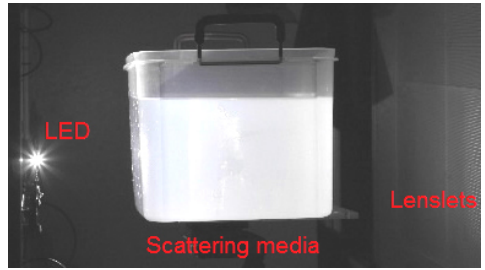

(a) Acutal Setup

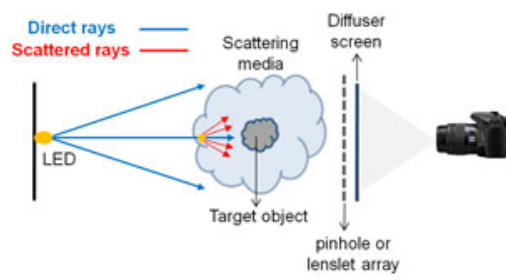

(b) Schematic Diagram

Fig. 2. (a) Imaging system consisting of an LED and a lenslet array. A single LED is used to back-illuminate a scattering scene. A diffuser is used to form an image through a lenslet array. A high-resolution camera captures the array of lenslet images in a single exposure. (b) A schematic diagram of the actual setup.

is required to recover a whole scene. Sheppard et al. [8] present a method to improve lateral and axial resolution. They achieve enhanced lateral resolution by subtracting a weighted traditional signal from a confocal imaging signal. Their method also increases axial resolution by using a number of detectors in different sizes. These are multi-exposure methods but are similar to our method where a simple linear transformation of intensities in a neighborhood recovers the sharp component. Levoy et al. 9] record 4D light field using a microlens array for digital refocusing and acquiring angular views with a single snapshot photo in microscope. In our method, the angular variation recorded by the similar way is exploited explicitly making it robust to non-homogeneous local variations. Our method requires no complicated light sources or mechanical scanning or change in aperture settings.

3D Recovery in Scattering Media: Narasimhan et al. 2. and $\mathrm{Gu}$ et al. 3] use sequential structured light patterns to recover 3D shape of static opaque objects in low density scattering media. Our method requires simple light sources and only a single photo per view. Atcheson et al. 4] recover non-stationary gas flows using Schlieren imaging and multiple cameras. The method is suitable for refracting but not scattering media. Rosen and Abookasis [6] proposed a method to recovery shape of binary objects between 2 layers of scattering media based on refocusing principles. Trifonov et al. [10] consider tomographic reconstruction of transparent objects using large number of photos and index matching liquids. Our emphasis is on scattering objects.

\section{Imaging System}

\subsection{Overview}

The actual setup and schematic diagram of our proposed imaging system is shown in Figure 2, We note that our direct-scattered separation method handles solid objects and liquid mixtures. In particular, we consider the case when a solid object is enclosed by a scattering media, as is typical in medical imaging 


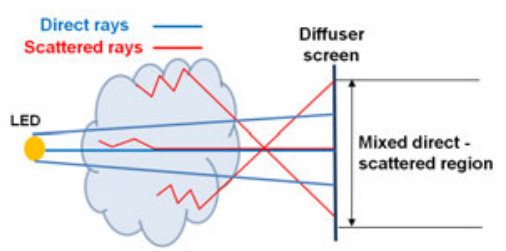

(a) Without any optical component

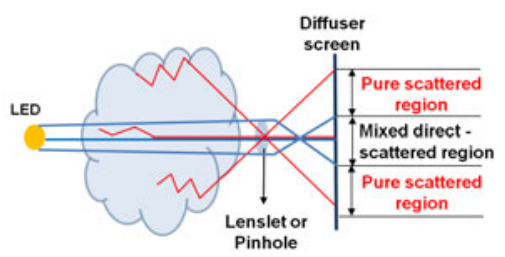

(b) With lenslet or pinhole

Fig. 3. Recovering the direct component from a mixed direct-scattered region. There is no way to separate direct and scattered rays in (a). The rays are spatially separated by a lenslet or pinhole in (b). We use the estimate from the pure-scattered region to subtract the scattered component in the central region.

applications. The property of the solid object can be most types of scattering except significant internal refraction. Refraction is treated as scattering and appropriately separated from direct component but the low frequency fitting of our method(RTE scattering model) becomes inaccurate. Thin glass objects and thin boundary of media with minor refraction are fine. Under a simple geometric scattering model, light rays are emitted from a point source (an LED in our system). When each direct ray impinges on a scattering center, a new scattering light source is effectively created(Figure 2(b)). Both direct and scattered rays form an image through a pinhole or lenslet array onto a diffuser screen which is captured by a camera. We apply radiative transport equation(RTE) [11] to model the angular variation of this scattering center. We assume, at a basic level, that the heterogenous scattering media will be dominated by multiple scattering events 12 ] 13 .

\subsection{Imaging with Lenslets or Pinhole Arrays}

We use a Canon EOS Digital Rebel XSi, with a resolution of $4272 \times 2848$ pixels. The lenslet array is separated from the diffuser in order to form an image of the scattering volume focusing on the entire volume with large DOF(centimeters almost infinite) of lenslet(Figure 2(b)). Lanman et al. 14] used a similar imaging setup to compute a single shot lightfield of opaque objects while we address scattering to compute direct component of translucent objects. From Figure 3(b), we infer there are two regions in the image under each lenslet. The first region consists of a mixed signal due to cross-talk between the direct and scattered components. The second region represents a pure scattered component. In the following section, we show a simple method for analyzing such imagery to separate direct and scattered components for multiple-scattering media. As shown in Figure 3(b), the angular sample directly under each lenslet can be used to estimate the combined direct plus scattered transmission along the ray between a given pixel and the light source. Similarly, any non-zero neighboring pixels(not beneath the lenslet) can be fully attributed to scattered illumination due to volumetric scattering. 

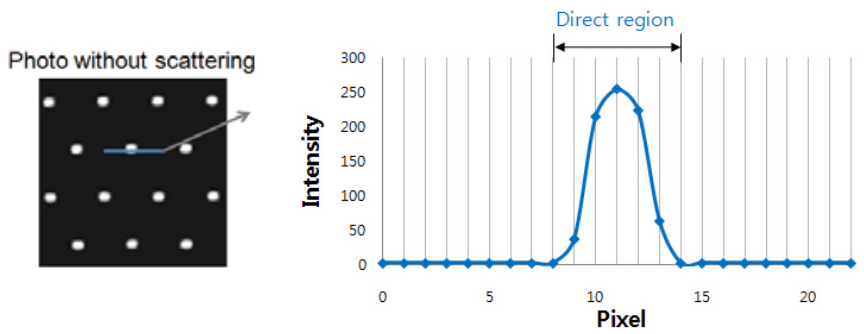

(a) Without Scattering
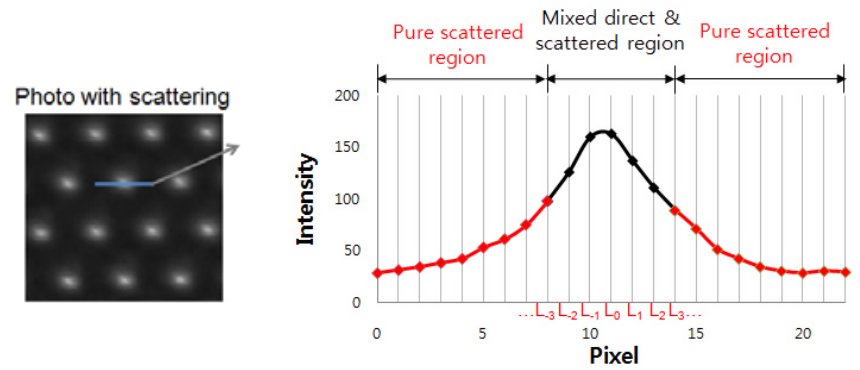

(b) With Scattering

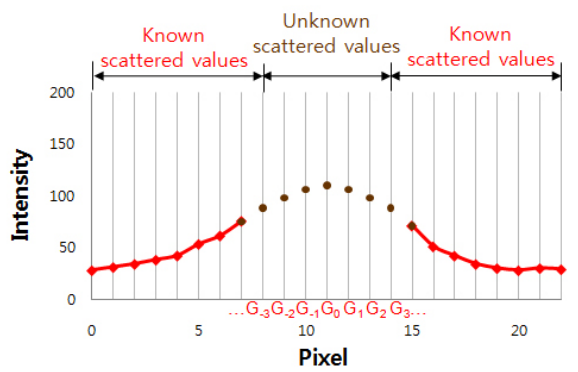

(c) Estimation

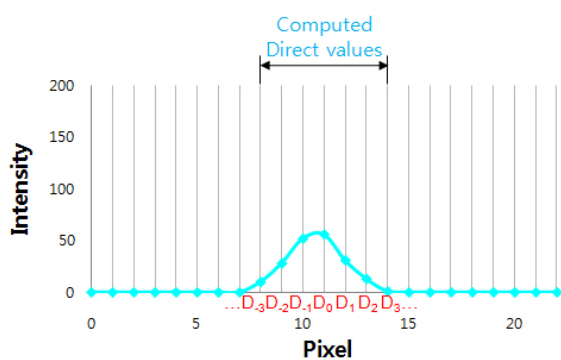

(d) Subtraction

Fig. 4. Comparison of direct and scatter components without and with scattering media. (a) Central region under each lenslet is sharp without scattering. (b) Direct as well as scattered component is included in the central region. (c) Measured (red) and estimated (brown) values for scattering-only component. (d) The direct-only image formed by subtracting (c) from (b). 


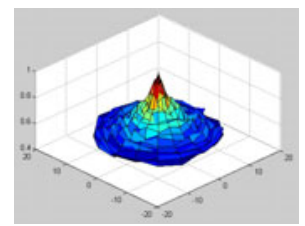

(a) Measured Data

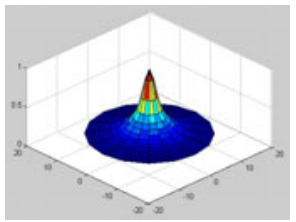

(e) $\mathrm{q}=0.9, \mathrm{~T}=2.0$

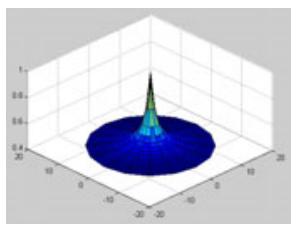

(b) $\mathrm{q}=0.9, \mathrm{~T}=1.4$

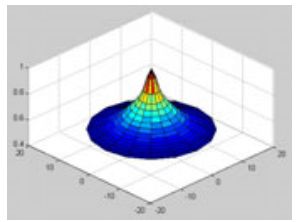

(f) $\mathrm{q}=0.2, \mathrm{~T}=2.0$

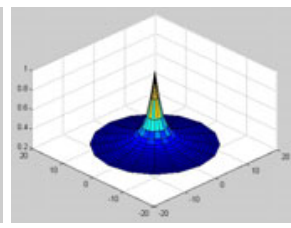

(c) $\mathrm{q}=0.7, \mathrm{~T}=1.4$

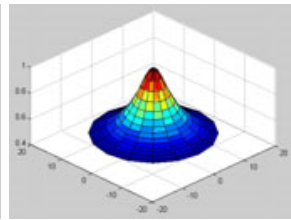

(g) $\mathrm{q}=0.9, \mathrm{~T}=4.0$

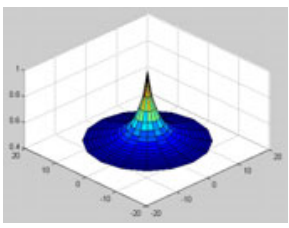

(d) $\mathrm{q}=0.2, \mathrm{~T}=1.4$

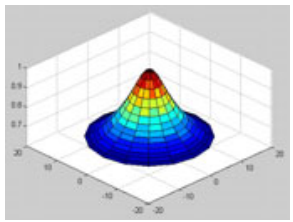

(h) $\mathrm{q}=0.2, \mathrm{~T}=4.0$

Fig. 5. RTE(Radiative Transport Equation) modeling of scattering values through a pinhole. (a) Measured data (b)-(h) RTE Modeling with different $q$ and T. (f) is in minimum fitting error with the measured data.

\section{Direct-Scattered Separation}

\subsection{Separation via Angular Filtering}

In this section we consider direct-scattered separation for a 1-D sensor and a 2-D scene, while the results can be trivially extended to 2-D sensors and 3-D volumes. In the following analysis, we consider only lenslet arrays, however a similar analysis holds for pinhole arrays. As shown in Figure 4(a), the diffuserplane image, a reference image to be captured in a calibrated setup, consists of a set of sharp peaks under each lenslet in the absence of any scattering media between the light source and diffuser. As shown on 4(b), the lenslet images contain extended, blurred patterns when a scattering object is placed between the light source and camera. Ultimately, the scattered light causes samples to appear in pixels neighboring the central pixel under each lenslet. A single lenslet image is defined by two separate regions: a pure scattered component region and a region of mixed direct and scattered components. We represent the received intensity at each diffuser-plane pixel as, $\left\{L_{0}, L_{1}, \ldots, L_{n}\right\}$, when a scattering object is placed between the light source and the diffuser. The individual sensor values are modeled as

$$
\begin{gathered}
L_{0}=G_{0}+D_{0} \\
\vdots \\
L_{n}=G_{n}+D_{n},
\end{gathered}
$$

where $\left\{G_{n}\right\}$ and $\left\{D_{n}\right\}$ represent the underlying scattered and direct intensities measured in the sensor plane, respectively. As shown in Figure 4(b), a straightforward algorithm can be used to estimate the direct and scattered components 


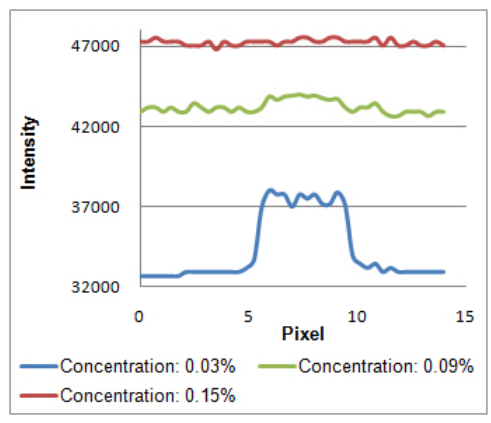

(a) Normal Photo

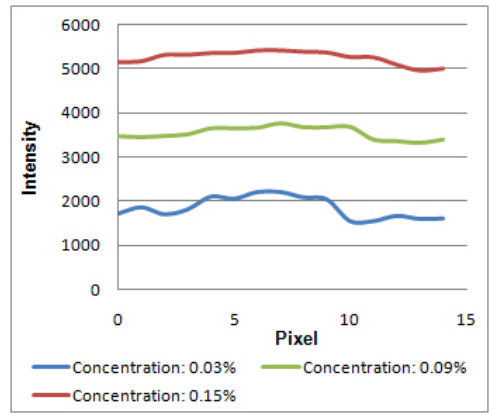

(c) Scattered-only Image

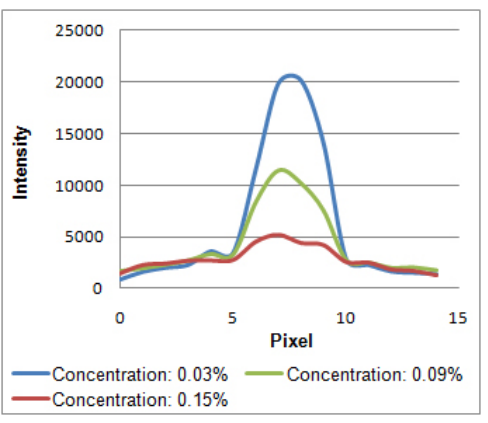

(b) Direct-only Image

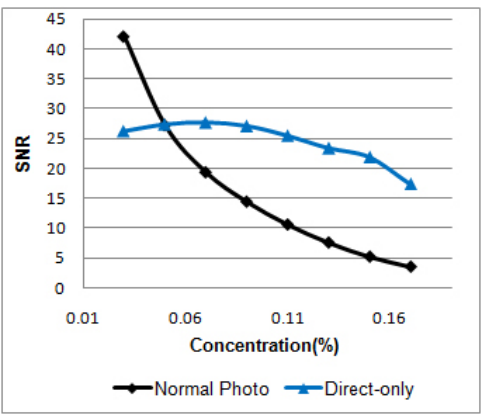

(d) SNR Comp.

Fig. 6. (a)-(c) Intensity profiles show that signals in direct-only images are significantly enhanced compared with those in normal photos(Intensity scale 0-65535) (d) SNR comparison between normal and direct-only images shows that our method is effective at scattering-dominent scene.

received at each lenslet. First, we estimate the non-zero region in (a) which is captured with no object present. Next, we approximate values of the scattering component $\left\{G_{n}\right\}$ in the region using a scattering model, described in next section, as shown in (c). Note that this region is subject to mixing in (b) and the scattering component must be approximated from the known scattered values in (c). Finally, a direct-only image can be estimated by subtracting the estimated scattering component for the central pixel under a lenslet, such that $D_{0} \approx L_{0}-G_{0}$.

\subsection{Mathematical Model for Multiple Scattering}

We describe the multiple scattering model used in the descattering algorithm described in the previous section. Numerical Monte-Carlo techniques have been widely used for tracing scattered rays but it needs high computational cost for a large number of rays. To implement efficient descattering algorithm, we use the physics-based model presented by Narasimhan and Nayar 11. Multiple scattered intensity through a pinhole can be described by RTE (Radiative Transport 

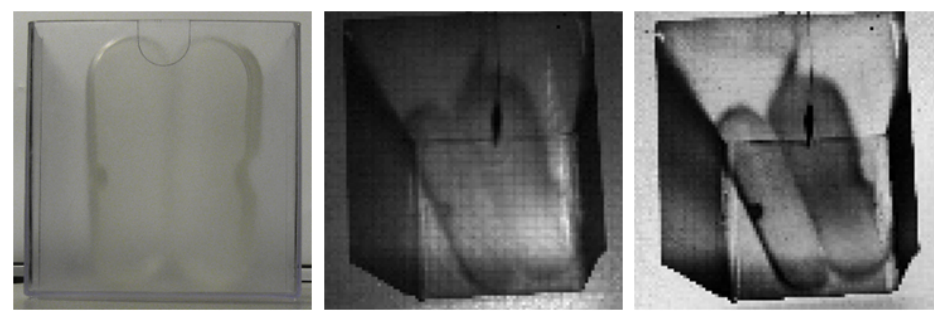

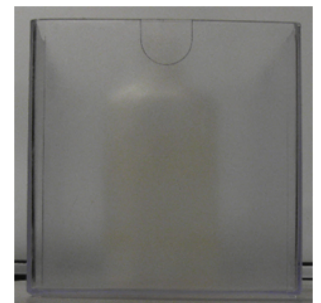

(a) Room-light Photo

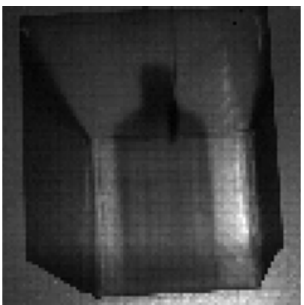

(b) Direct+Scattered with a backlight

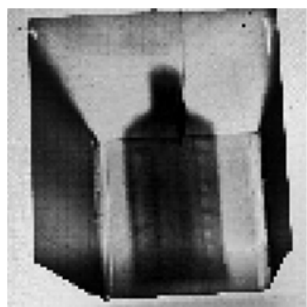

(c) Direct-only

Fig. 7. Direct-scattered separation with a pinhole array. Direct-only images enhance high frequency features of an object enclosed by a scattering solid object.

Equation) and the solution of it is a function of three parameters, $\mathrm{T}$ (optical thickness), $\mathrm{q}$ (forward scattering parameter) and $\mathrm{x}$ (spatial position) as explained in the Narasimhan and Nayar's paper. RTE is used to fit measured 2D data, Figure 5(a), under each lenslet of our imaging condition. (b)-(h) show varied intensity distributions according to different $\mathrm{T}$ and $\mathrm{q}$. By an iterative error-minimization method, the best matching profile, (f), can be found for the measured 2D signal (a) and any unknown scattered value for nearby regions can be approximately calculated by the fitted model.

\subsection{Experimental Results}

From Section 3.1, we separate direct signals $\left\{D_{n}\right\}$ and scattered signals $\left\{G_{n}\right\}$ in each lenslet region. By collecting and combining the direct signals in each lenslet (or pinhole) region, we can generate a direct image. The scattered image is obtained by a similar process, collecting scattered signals. The original image(or normal photo) can be considered as the summed image of the direct and scattered signals. The resolution of the direct and scattered component images are identical to the number of lenslets (or pinholes), because there is only one signal value for direct and scattered components for each lenslet region. In our experiment, the image size is $150 \times 100$.

Figure 1 1 compare normal photos of a scattering scene, consisting of an opaque horse-shape object enclosed in an aquarium with milky water, and direct-only images generated by our proposed separation process in lenslet array setup. From left to right, the images show results acquired at higher concentrations of milky water. Figure 6 (a)-(c) compare signals at the position of the red line 


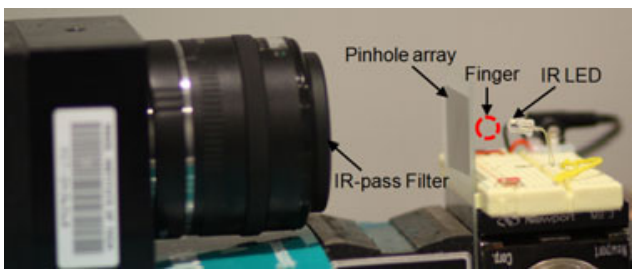

(a) IR Imaging Setup
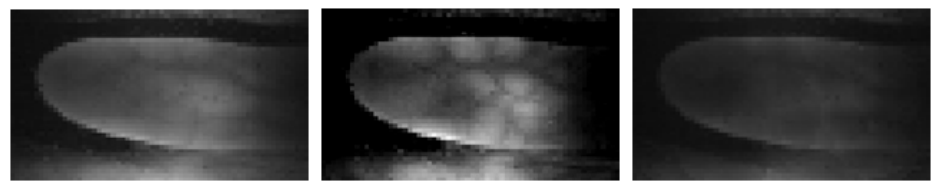

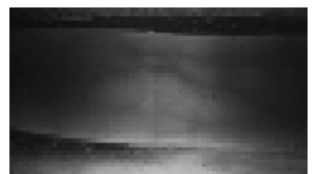

(b) Normal Photo

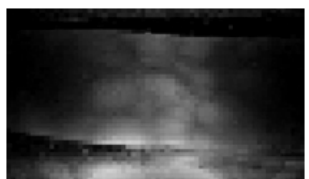

(c) Direct-only

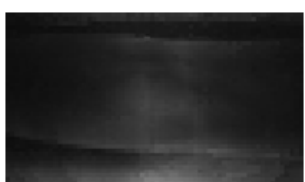

(d) Scattered-only

Fig. 8. Direct-scattered separation images for human fingers using infrared imaging setup. Direct-only images show sharper shapes of finger veins than normal photos. (a) The camera focuses on the pinhole array plane. The IR-pass filter cut visible light and only pass IR light.

in Figure 1(a) for normal photos, direct-only images and scattered only images at different concentrations. (b) shows the signals are enhanced compared with signals in normal photos, (a). As the concentration of milky water is increased, the intensity of the signal in direct-only images, (b), is decreased. The opposite effect is observed in scattered-only images, (c), which follows physical reasoning. (d) compares the signal-to-noise ratio(SNR) between normal photos and direct-only images according to concentration. At low concentration, the SNR of a normal photo is larger than one of a direct-only image. However, as concentration is increased, the SNR of a direct-only image gradually becomes higher than the SNR of a normal photo. Note that the signal of normal photos, (a), is significantly decreased from the concentration $0.03 \%$ to $0.09 \%$ compared with the signal change in direct-only images in (b).

Figure 7 shows experimental results using a pinhole array instead of a lenslet array. (a) shows the room-light photo of a solid object placed in the scattering medium. (b) displays ground-truth photos which are acquired by summing all direct and scattered values under each lenslet. (c) contains the direct-only image. By comparing (b) and (c), we find that the direct-only images give the sharpest image boundaries for the scattering objects.

We tested our method for human fingers with a near-infrared imaging setup where finger veins are well visualized with infrared light. Direct-only images in Figure 8 (c) shows sharper shape of the veins than normal photos do. This is an initial experiment for a strongly scattering challenging object but the image 


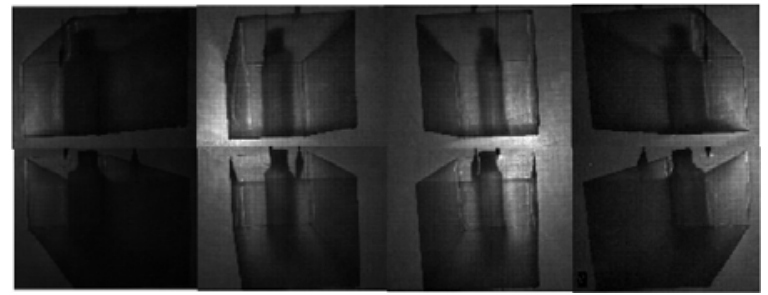

(a) Normal Photo

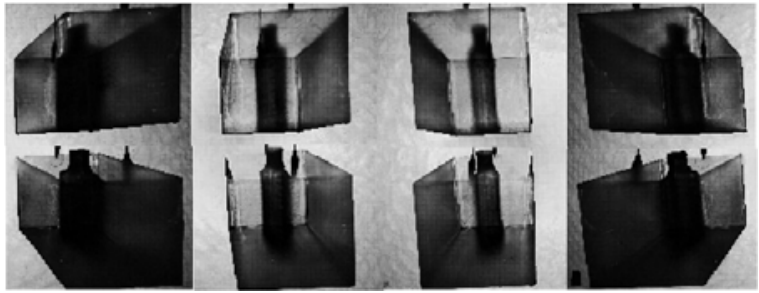

(c) Direct-only images

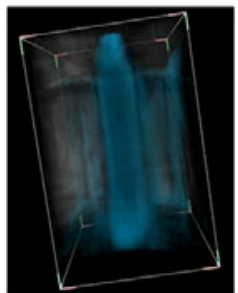

(b) $3 \mathrm{D}$ result

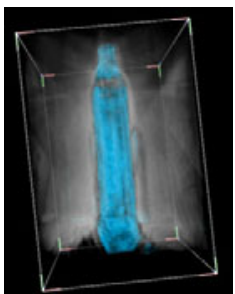

(d) $3 \mathrm{D}$ result

Fig. 9. Tomographic reconstruction resutls. (a) and (b) show eight normal photos captured at different light-projection angles and 3D result using them, respectively. (c) and (d) are direct-only images for the each normal photo and 3D result using the direct-only images.

formation model is identical to Figure 1 and our results are comparable to [15] which uses specialized narrow wavelength profile light source. As in Figure1 veins closer to the skin are more prominently visible as they are decomposed in the direct component although the finger is strongly scattering. Such enhanced visualization of a human body will benefit medial, biometrics and HCI applications.

\subsection{Volumetric Reconstruction Using ART}

We use an algebraic reconstruction technique (ART) presented by Roh et al. [16] to reconstruct 3-D shape of scattering objects following traditional short-baseline tomography approaches. Figure 9(b) and (d) compare two 3-D reconstruction results using eight normal photos and eight descattered images. We captured eight photos sequentially with a LED mounted at different position to get multiple angular views of the targeted inside object, the bottle, in Figure 7 (bottom). In the 3D reconstruction, Figure 9(b), the bottle is rendered by blue color to add distinguishability from the outer scattering container. Note that the rendering isn't accurate for the bottle since the bottle shape in the captured images has been hazed by scattering. The 3D result using direct-only images in (d) shows a more accurate rendering result for the inside bottle object.

\section{Benefits and Limitations}

Benefits: This paper makes three primary contributions: (1) robust separation of direct and scattered components of incident light passing through heterogenous 


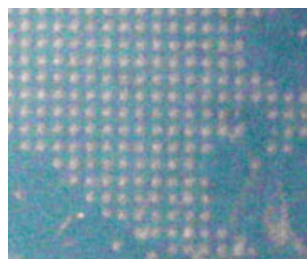

(a) Inset Photo

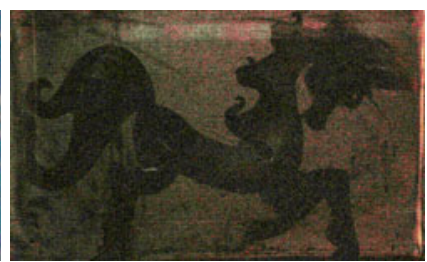

(b) direct-only(wide DOF)

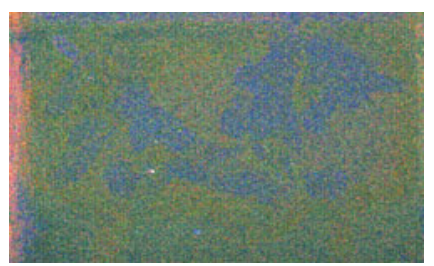

(c) direct-only(short DOF)

Fig. 10. Direct-only images by Nayar et al. [1] method for a horse-shaped object enclosed in an aquarium with diluted milk(Concentration $0.11 \%$ ) (a) Inset of a captured photo with a projected high-frequency pattern (b) Direct-only image with wide projector's DOF (c) Direct-only image with short projector's DOF

scattering media, (2) 3-D volumetric reconstruction of the mixed scattering objects, and (3) a novel technique to enable effective volumetric analysis of solid scattering objects in multiple-scattering conditions. For direct and scattered separation, our method requires only a simple system consisting of a lenslet(or pinhole array) and a point light source. Compared with other methods, like using a projector to generate temporally-multiplexed patterns, our method can achieve separation in a single exposure. Also, our method requires simple local computations, performed independently on each lenslet image. Furthermore, dynamic applications are possible.

Our 3-D reconstruction technique for scattering objects has potential applications extending beyond computer vision and graphics, including non-invasive medical imaging [17. Specific parts of biological organisms, including human fingers, can be modeled by scattering and translucent material similar to objects considered in this work. As a result, it is possible to view the inner 3-D shape of certain parts in human and animal bodies by this technique. Most importantly, such visible-wavelength separation methods may allow hazardous X-ray imaging to be replaced in certain applications. Such applications include the personal identification field. For example, 3-D shape recognition of finger veins can provide strong cues for identification. Furthermore, such features may overcome several limitations of traditional fingerprints, which change due to aging.

For transmission-mode descattering, the proposed method has several unique advantages in comparison to the closely related method of Nayar et al. 1]. One of the key limitations of Nayar et al. [1] is that the assumption of high-frequency projected patterns aren't satisfied in dense media(Figure 10 (a)). Another limitation of any projector-based solution, such as that of Nayar et al., arises due to the finite DOF(Depth of Field) achieved in practice. For transmission-mode descattering, the projector must focus on the scattering media and the screen at the same time - unlike the case of reflection-mode acquisition. Thus, the projector requires a wide DOF. Figure [10(c) shows a direct image by [1] when the projector's DOF isn't wide enough to cover both inside object and screen. Our proposed method is free from such focusing problems. Furthermore, our proposed 


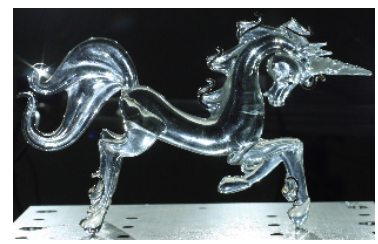

(a) Room Light Photo

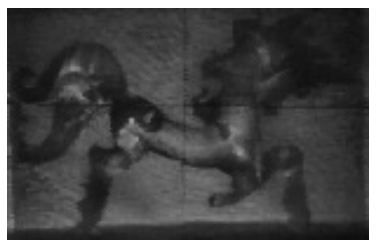

(b) Direct-only

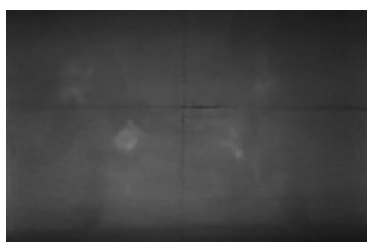

(c) Scattered-only

Fig. 11. Limitation of refraction. Our method results for a translucent horse-shaped object enclosed in an aquarium with diluted milk(Concentration $0.11 \%$ ).

imaging system is much simpler and inexpensive, containing a single LED. Finally, our method is well-suited for less dense parts of human bodis, such as fingers as shown in Figure 8

Limitations: In the current method, the primary limitation is due to the loss of resolution incurred by the lenslet or pinhole array. In addition, pinhole arrays require long exposures. While lenslets could be used to overcome exposure issues, loss of resolution remains. Also, the separated results can be affected by refraction. Figure 11 shows separated results of a translucent horse-shape object in milky water. Note that the legs and the end of the tail in (b) look dark by refraction although they have similar density with the body area as shown in (a). The proposed 3D reconstruction method requires control of the lighting environment and, as a result, cannot be directly extended to natural environments. Furthermore, this reconstruction method requires a temporallymultiplexed set of images for tomographic reconstruction, limiting dynamic scene reconstruction. We emphasize, however, that direct-scattered separation can be performed in a single exposure. Most importantly, we anticipate challenges in strongly-scattering environments. In such circumstances, the scattering term will dominate the proposed low-order polynomial approximation and the direct term will not be reliably recovered.

\section{Conclusion}

In this paper, we show a new method to separate direct and scattered components of transmitted light from translucent objects. The direct-only images provide sharp shape information for such scattering objects. We have demonstrated a volumetric reconstruction technique, following classic methods of limitedbaseline tomography, to reconstruct scenes using direct-only images. These results can be achieved with low-cost hardware consisting of LEDs, diffusers, and lenslet array(or printed pinhole array mask). In particular, we show that visiblewavelength radiation can be applied for attenuation-based tomography when such separation methods exist in the transmission-mode. We hope that our research will inspire others to pursue low-energy, non-invasive imaging in the medical and biological sciences. 


\section{Acknowledgments}

Ramesh Raskar is supported by an Alfred P. Sloan Research Fellowship.

\section{References}

1. Nayar, S., Krichnan, G., Grossberg, M., Raskar, R.: Fast separation of direct and global components of a scene using high frequency illumination. In: ACM TOG, vol. 12, pp. 935-943 (2006)

2. Narasimhan, S.G., Nayar, S.K., Sun, B., Koppal, S.J.: Structured light in scattering media. In: Proc. IEEE ICCV, vol. 1, pp. 420-427 (2005)

3. Gu, J., Nayar, S., Grinspun, E., Belhumeur, P., Ramamoorthi, R.: Compressive structured light for recovering inhomogeneous participating media. In: Forsyth, D., Torr, P., Zisserman, A. (eds.) ECCV 2008, Part IV. LNCS, vol. 5305, pp. 845-858. Springer, Heidelberg (2008)

4. Atcheson, B., Ihrke, I., Heidrich, W., Tevs, A., Bradley, D., Magnor, M., Seidel, H.P.: Time-resolved 3D capture of non-stationary gas flows. In: ACM TOG (2008)

5. Nasu, O., Hiura, S., Sato, K.: Analysis of light transport based on the separation of direct and indirect omponents. In: PROCAMS (2007)

6. Rosen, J., Abookasis, D.: Noninvasive optical imaging by speckle ensemble. Optics Letters 29 (2004)

7. Okugawa, H.: A new imaging method for confocal microscopy. In: SPIE (2008)

8. Sheppard, C.J.R., Cogswell, C.J.: Confocal microscopy with detector arrays. Journal of Modern Optics 37, 267-279 (1990)

9. Levoy, M., Zhang, Z., Mcdowall, I.: Recording and controlling the $4 \mathrm{~d}$ light field in a microscope using microlens arrays. J. of Microscopy (2009)

10. Trifonov, B., Bradley, D., Heidrich, W.: Tomographic reconstruction of transparent objects. In: Eurographics Symposium on Rendering (2006)

11. Narasimhan, S.G., Nayar, S.K.: Shedding light on the weather. In: Proc. IEEE CVPR, vol. 151, pp. 665-672 (2003)

12. Jensen, H., Marschner, S., Levoy, M., Hanrahan, P.: A practical model for subsurface light transport. In: SIGGRAPH, pp. 511-518 (2001)

13. Sun, B., Ramamoorthi, R., Narasimhan, S.G., Nayar, S.K.: A practical analytic single scattering model for real time rendering. In: TOG, pp. 1040-1049 (2005)

14. Lanman, D., Raskar, R., Agrawal, A., Taubin, G.: Shield fields: Modeling and capturing 3d occluders. In: SIGGRAPH Asia 2008 (2008)

15. Miura, N., Nagasaka, A.N., Miyatake, T.: Feature extraction of finger-vein patterns based on repeated line tracking and its application to personal identification. Machine Vision and Applications (2004)

16. Roh, Y.J., Park, W.S., Cho, H.S., Jeon, H.J.: Implementation of uniform and simultaneous ART for 3-D reconstruction in an x-ray imaging system. In: IEEE Proceedings, Vision, Image and Signal Processing, vol. 151 (2004)

17. Tuchin, V.: Tissue Optics: Light Scattering Methods and Instruments for Medical Diagnosis. SPIE Publications (2007) 